\title{
A simple test for the cleavage activity of customized endonucleases in plants
}

\author{
Nagaveni Budhagatapalli ${ }^{1 \dagger}$, Sindy Schede ${ }^{1 \dagger}$, Maia Gurushidze ${ }^{1}$, Stefanie Pencs ${ }^{1,2}$, Stefan Hiekel ${ }^{1}$, Twan Rutten ${ }^{3}$, \\ Stefan Kusch ${ }^{4}$, Robert Morbitzer ${ }^{5}$, Thomas Lahaye ${ }^{5}$, Ralph Panstruga ${ }^{4}$, Jochen Kumlehn ${ }^{1}$ and Goetz Hensel ${ }^{1^{*}}$
}

\begin{abstract}
Background: Although customized endonucleases [transcription activator-like effector nucleases (TALENs) and RNA-guided endonucleases (RGENs)] are known to be effective agents of mutagenesis in various host plants, newly designed endonuclease constructs require some pre-validation with respect to functionality before investing in the creation of stable transgenic plants.
\end{abstract}

Results: A simple, biolistics-based leaf epidermis transient expression test has been developed, based on reconstituting the translational reading frame of a mutated, non-functional yfp reporter gene. Quantification of mutation efficacy was made possible by co-bombarding the explant with a constitutive $m$ Cherry expression cassette, thereby allowing the ratio between the number of red and yellow fluorescing cells to serve as a metric for mutation efficiency. Challenging either stable mutant alleles of a compromised version of gfp in tobacco and barley or the barley MLO gene with TALENs/RGENs confirmed the capacity to induce site-directed mutations.

Conclusions: A convenient procedure to assay the cleavage activity of customized endonucleases has been established. The system is independent of the endonuclease platform and operates in both di- and monocotyledonous hosts. It not only enables the validation of a TALEN/RGEN's functionality prior to the creation of stable mutants, but also serves as a suitable tool to optimize the design of endonuclease constructs.

Keywords: Transcription activator-like effector nucleases, RNA-guided endonucleases, Biolistic gene transfer, Site-directed mutagenesis, Transient expression

\section{Background}

The exploitation of either customizable transcription activator-like effector nucleases (TALENs) [1,2] or RNAguided endonucleases (RGENs) [3, 4] has opened up numerous possibilities for site-directed mutagenesis and precise genome editing in plants. The gaps induced in the host's DNA are repaired by either non-homologous end joining or by homology-directed repair. The former process is error-prone and so randomly introduces insertions and deletions (indels), while the latter, which exploits the respective locus of the sister chromatid or an artificially

\footnotetext{
*Correspondence: hensel@ipk-gatersleben.de

${ }^{\dagger}$ Nagaveni Budhagatapalli and Sindy Schedel contributed equally to this work

${ }^{1}$ Plant Reproductive Biology, Leibniz Institute of Plant Genetics and Crop Plant Research (IPK), Corrensstr. 3, 06466 Stadt Seeland/OT Gatersleben, Germany

Full list of author information is available at the end of the article
}

provided DNA that combines homology to the target site with an alteration of choice as repair template, is highly precise $[5,6]$. The documented use of customized endonucleases in plants to date has largely involved Arabidopsis thaliana, Nicotiana benthamiana, maize or rice [7]. A few inherited TALEN- or RGEN-induced mutations have been reported in both barley [8,9] and wheat [10]. Budhagatapalli et al. [11] have demonstrated the feasibility of homology-directed editing of barley at the cellular level. In mammalian cells, mutation frequencies of up to, respectively, 60 and $80 \%$ have been achieved following the application of TALENs and RGENs [12].

Customizing endonucleases remains a somewhat empirical process, which would benefit from the development of a simple validation assay able to be carried out prior to transformation. The purpose of the present research was to establish a chimeric expression construct 
comprising a target sequence positioned upstream of a mutated, non-functional copy of $y f p$ (encoding the cellular marker yellow fluorescent protein). The compromised $y f p$ sequence features a frameshift mutation which can be reversed by the activity of a sequence-specific endonuclease; thus the frequency of cells producing YFP was expected to reflect the cleavage activity of the endonuclease. The assay has been deliberately designed to be usable with any customizable endonuclease platform in both diand monocotyledonous plant species. One of the chosen targets in barley was the $M L O$ gene, the product of which is associated with susceptibility to infection by Blumeria graminis, the causative fungal pathogen of powdery mildew; mlo alleles, which fail to produce a functional MLO protein, typically imbue the host with a durable, broadspectrum resistance against the disease $[13,14]$.

\section{Results}

The test system in the dicotyledonous species tobacco

The principle of the assay is that the error-prone repair of endonuclease-induced double-strand breaks would generate indels at the target site. Consequently, a proportion of induced mutations were expected to restore the functionality of the compromised $y f p$ sequence positioned downstream of the target sequence (Fig. 1). When tobacco (Nicotiana tabacum cv. SR1) leaf segments were co-bombarded with a nuclease-specific vector construct along with a TALEN or an RGEN construct, a number of epidermal cells began to accumulate YFP (Fig. 2a). The mutation frequency was quantified by cobombarding a constitutive $m$ Cherry expression cassette; the ratio between the number of red and yellow fluorescing cells was then used to estimate the efficiency of the endonuclease construct (Fig. 2b). The co-bombardment of pTARGET-gfp1 with the pair of $g f p$-specific TALEN constructs (Table 1) generated $100 \pm 71$ yellow and $363 \pm 148$ red fluorescent cells, corresponding to a ratio of $27 \%$; meanwhile, the co-bombardment of pTARGETgfp1 with the $g f p$-specific RGEN construct yielded a ratio of $75 \%$ (Table 2). Bombardment with the pTARGET-gfp1 vector on its own failed to induce any cells to synthesize YFP.

To estimate the frequency of mutations achievable in a stably transformed plant, a transgenic tobacco plant harboring a single copy of $g f p$ [15] was co-transformed with the pair of $g f p$-specific TALEN constructs to produce plants carrying only one of the TALEN units; no mutations were detectable in the $g f p$ sequence in either transgenic. The two TALEN units were then brought together by intercrossing the primary transgenics. Of the 35 progeny found to harbor both $g f p$-specific TALEN units (Table 3 ), one carried a mutated form of $g f p$. In a similar experiment based on the $g f p$-specific RGEN construct, 17 transgenics were obtained; of these, 15 carried Cas 9 and guide-RNA (gRNA), and of these, 12 contained mutations (Table 3 ). The mutations obtained were diverse in nature [15].

\section{The test system in the monocotyledonous species barley}

Co-bombardment of barley (Hordeum vulgare cv. 'Golden Promise') leaves with pTARGET-gfp1 and the pair of $g f p$-specific TALEN constructs (Table 1) produced $77 \pm 25$ epidermal cells showing yellow (YFP) fluorescence and $250 \pm 59$ exhibiting red (mCherry) fluorescence (Table 2), yielding a ratio of $30.7 \%$. Bombardment with the $g f p$-specific RGEN construct and pTARGET-gfp2 led to a relative cleavage activity of $31.4 \%$. To validate the functionality of TALENs targeting an endogenous barley gene, a further co-bombardment of the same barley material was conducted using the $M L O$-specific pTARGET-MLO reporter plasmid and the appropriate TALEN pair \#3: this resulted in a relative cleavage activity of $42.0 \%$ (Table 2 ). The $M L O$-specific TALEN constructs were also tested in combination with the GUS reporter gene in barley (cv. 'Ingrid'; using bombardment) and in N. benthamiana (using Agrobacterium tumefaciens-mediated transient expression) using five different TALEN pairs targeting two different exons of the $M L O$ gene. Out of the five tested TALEN pairs (Additional file 1), only TALEN pairs \#2 and \#3 showed detectable activity and restored GUS expression when the TALEN constructs were co-transformed with the respective reporter construct (Additional file 2). While TALEN pair \#2 yielded only very few GUS-positive cells in barley and $N$. benthamiana, TALEN pair \#3 was more active and reproducibly led to GUS-positive cells in both plant systems (Additional file 3). Note that in the case of this assay, there was no means of normalizing the transformation efficiency due to the lack of a second reporter.

To assess the efficacy of the TALEN constructs in a stably transformed barley line, a transgenic version of cv. 'Igri' harboring $g f p$ [8] was re-transformed. TALENinduced mutations in $g f p$ were detected in four out of $66 \mathrm{~T}_{0}$ plants (Table 3; Fig. 3). The induced mutations included various deletions in the size range of 15-172 nt. Therefore, one can conclude mono-allelic mutations for the $g f p$ gene. When the $M L O$-specific TALEN construct was transformed into cv. 'Golden Promise', three of the six regenerants included a deletion in $M L O$ : one harbored a single 15 nt deletion, while clones derived from the other two harbored a range of 4-8 nt deletions (Fig. 4). While two plants were considered bi-allelic mutants, in one $M L O$ mutant plant, also wild-type alleles were detectable leading to the conclusion of a mono-allelic alteration. 


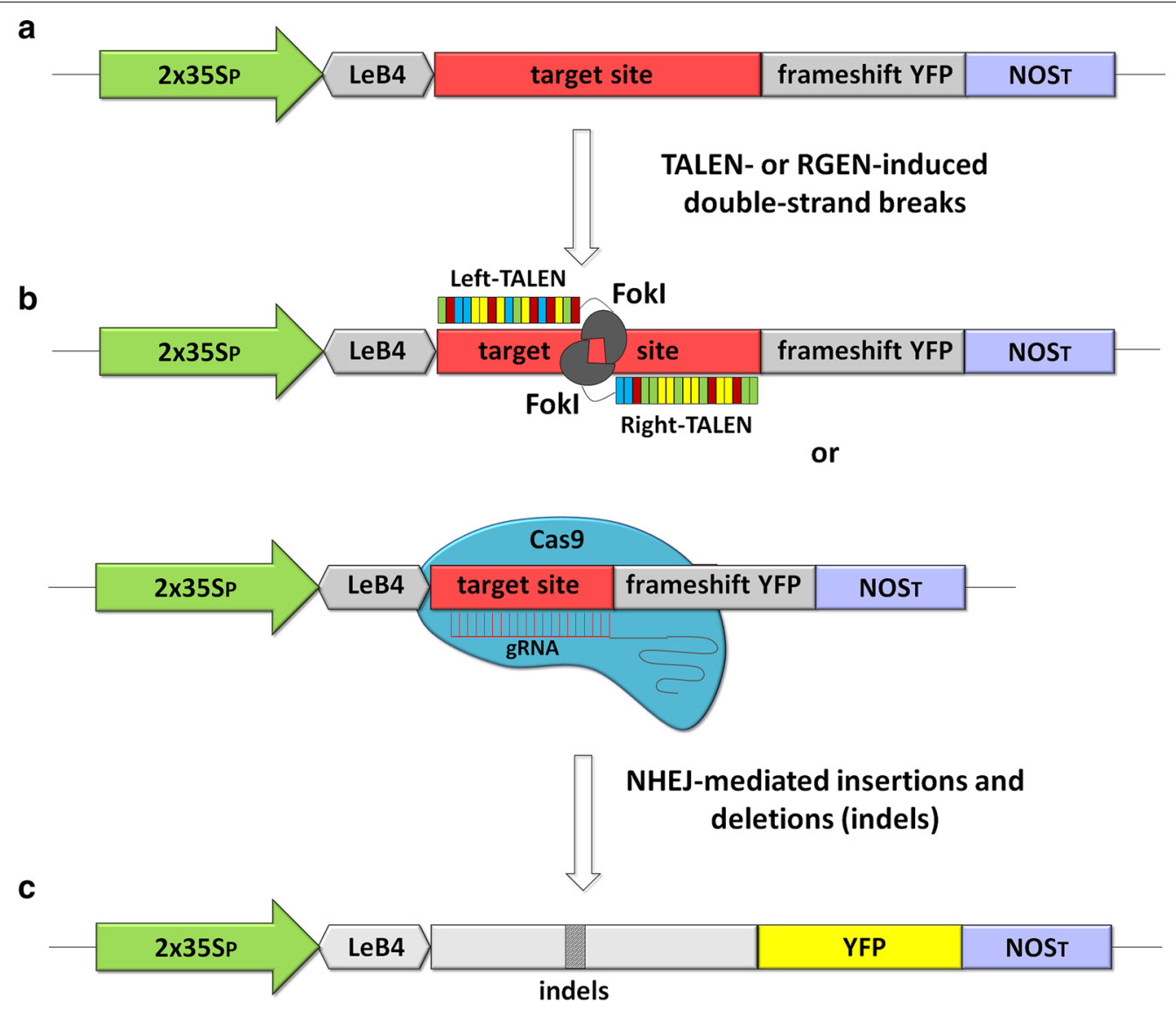

Fig. 1 The principle of the transient expression system used to assess the relative cleavage activity of customized endonucleases. a The incorporation of a target site for sequence-specific endonucleases generates a frame shift in the yfp sequence. $\mathbf{b}$ Upon co-transformation of the target vector with TALENs or RGENs, double- strand DNA breaks at the target site are induced. c The imperfect repair of these breaks via non-homologous end-joining can restore the wild type reading frame, thereby leading to expression of yfp and the emission of a YFP signal. The elements shown are not drawn to scale. 2×35SP: doubled enhanced CaMV 35 S promoter; LeB4: Vicia faba legumin B4 signal peptide; YFP: synthetic yellow fluorescent protein gene; NOST: A. tumefaciens NOPALINE SYNTHASE termination sequence; Fokl: DNA cleavage domain of Flavobacterium okeanokoites type IIS restriction endonuclease; gRNA: guide-RNA; Cas9: Streptococcus pyogenes Cas9; TALEN: transcription activator-like effector nucleases; NHEJ: nonhomologous end joining

\section{Discussion}

Although endonuclease-enabled site-directed mutagenesis is known to be effective in a range of plant species [7], the tools presently available to aid the in silico design of binding modules (Target Finder [16], Talvez and Storyteller [17, 18], and TALgetter [19]) or gRNAs (CRISPR design [20], CRISPRer [21] and Deskgen [22]) do not consistently produce the desired outcome [23]. There is thus clearly potential for optimization based on reshuffling the current endonuclease systems, while entirely novel systems are also emerging (such as the Cpf1, see [4]). Here, a convenient platform for detecting the cleavage activity of an endonuclease construct was elaborated, based on the restoration of function to a compromised version of $y f p$, a gene which encodes the readily assayable yellow fluorescent protein. The principle of customized endonuclease-induced restoration of reporter gene function has been used previously in the context of transient expression via infiltration of leaves using Agrobacterium, which is amenable only for a limited number of plant species $[24,25]$. By contrast, the method presented here relies on particle bombardment and can thus be readily adopted in any plant species. An additional novel feature which was included was co-transformation with $m$ Cherry, in order to allow for the comparative quantification of mutation frequency induced by the TALEN/ RGEN construct. The concept of using two fluorescent reporters is related to a previously established assay system designed to assess the efficiency of gene silencing constructs [26].

The tobacco TALEN transient expression experiment established that around one third of the cells showing red 

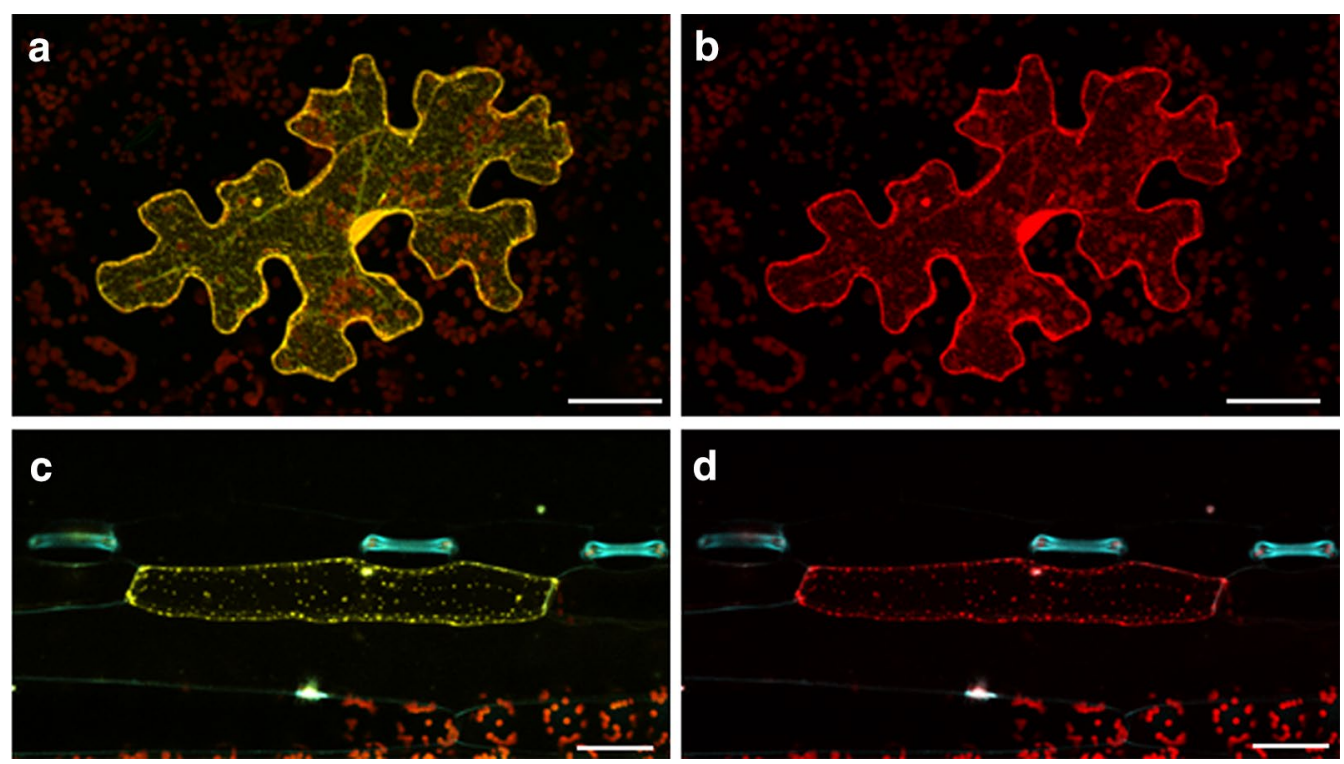

Fig. 2 Induced mutations as detected by yfp expression. Representative epifluorescing transgenic $\mathbf{a}, \mathbf{b}$ tobacco and $\mathbf{c}$, $\mathbf{d}$ barley cells visualized 1 day after bombardment with a nuclease-specific vector together with a TALEN or RGEN; $m$ Cherry was co-transformed to allow quantification. Bar $50 \mu m$

Table 1 List of TALEN and RGEN target site sequences and experiments used

\begin{tabular}{|c|c|c|c|}
\hline Construct & Target sequence & Species & Approach (constructs) \\
\hline \multirow[t]{3}{*}{ pTARGET-gfp1 } & \multirow[t]{3}{*}{ TGGTGAACCGCATCGAGCTGAAGGGCATCGACTTCAAGGAGGACGGCAAGT } & Tobacco & $\mathrm{RGEN}^{\mathrm{t} \text {, st }}(\mathrm{pSI} / 24)$ \\
\hline & & & TALEN $^{t, \text { st }}(p S P 10$, pSP11) \\
\hline & & Barley & TALEN $^{t, s t}(p G H 297, p G H 400)$ \\
\hline pTARGET-gfp2 & GTCTTTGCTCAGGGCGGACTGGG & Barley & RGEN $^{t}(\mathrm{pSH} 92)$ \\
\hline MLO pair \#1 & TGGTGCTCGTGTCCGTCCTCATGGAACACGGCCTCCACAAGCTCGGCCATGTA & Tobacco/barley & TALEN $^{t}(p 110 / 111)$ \\
\hline MLO pair \#2 & TCCTCATGGAACACGGCCTCCACAAGCTCGGCCATGTAAGTCCCGTTACCCTA & & TALEN $^{t}(p 112 / 113)$ \\
\hline MLO pair \#3 & TGGAACACGGCCTCCACAAGCTCGGCCATGTAAGTCCCGTTACCCTAGCTCAA & & TALEN $^{t}(p 114 / 115)$ \\
\hline MLO pair \#4 & TGCTGGCTTTGTATGCAGATGGGATCAAACATGAAGAGGTCCATCTTCGACGA & & TALEN $^{t}(p 124 / 125)$ \\
\hline MLO pair \#5 & TGGCTTTGTATGCAGATGGGATCAAACATGAAGAGGTCCATCTTCGACGAGCA & & TALEN $^{t}(\mathrm{p} 126 / 127)$ \\
\hline PTARGET-MLO & GCTGGAACACGGCCTCCACAAGCTCGGCCATGTAAGTCCCGTTACCCTAGCTCA & Barley & TALEN" ${ }^{t, s t}(p 114 / 115)$ \\
\hline
\end{tabular}

$t$ transient, st stable transgenic plants

fluorescence ( $m$ Cherry expressing) also generated a YFP signal (Fig. 2; Table 2), while this frequency was raised to about $75 \%$ when the RGEN construct was assayed (Table 2). The higher success rate achieved with the RGEN construct accords with the literature, and has been at least partly explained by the prediction that in contrast to TALENs, which exhibit a broad range of mutational events, most of the mutations induced by RGEN activity are $1 \mathrm{nt}$ insertions $[27,28]$. Consequently, the three possible reading frames of the reporter gene are unlikely to occur in balanced proportions in the latter platform. In the case of the $g f p$-RGEN approach in tobacco, the preferentially occurring insertion of one nucleotide restores the $y f p$ open-reading-frame and therefore, the comparatively high score achieved here is in accordance with the literature. By contrast, the $g f p$-RGEN approach in barley uses a different target site and in this case frame shifts by $1 \mathrm{nt}$ back or $2 \mathrm{nt}$ forward cause a functional $y f p$, which is not expected to occur as frequently as the shift by $1 \mathrm{nt}$ forward. In general, it is clear that comparisons of the efficacy of multiple nucleases based on a single target gene are only possible if the same frame-shift is used for the $y f p$ coding sequence succeeding the respective target motifs.

The induction of mutations to $g f p$ induced by stably incorporated TALEN and RGEN constructs corroborated the behavior shown in the transient expression assays. While only a low mutation frequency $(2.9 \%)$ was 
Table 2 Relative cleavage activity of RGEN and TALEN constructs in transiently transformed barley and tobacco leaf explants

\begin{tabular}{|c|c|c|c|c|c|c|c|}
\hline Plant species & Target gene & $\begin{array}{l}\text { Type of customized } \\
\text { endonuclease }\end{array}$ & Constructs used & Experiment & YFP cells & $\begin{array}{l}\text { mCherry } \\
\text { cells }\end{array}$ & $\begin{array}{l}\text { Ratio YFP/mCherry } \\
\text { cells (\%) }\end{array}$ \\
\hline \multirow[t]{12}{*}{ Tobacco } & $g f p$ & RGEN & pTARGET-gfp1 & 1 & 273 & 371 & 73.6 \\
\hline & & & + RGEN-gfp & 2 & 342 & 389 & 87.9 \\
\hline & & & & 3 & 324 & 499 & 64.9 \\
\hline & & & & Average & $313 \pm 29$ & $420 \pm 57$ & 74.6 \\
\hline & & & pTARGET-gfp1 & 1 & 0 & 106 & 0 \\
\hline & & & & 2 & 0 & 204 & 0 \\
\hline & & & & 3 & 0 & 81 & 0 \\
\hline & & & & Average & 0 & $130 \pm 65$ & 0 \\
\hline & & TALEN & pTARGET-gfp1 & 1 & 195 & 361 & 54.0 \\
\hline & & & + TALEN-gfp & 2 & 25 & 183 & 13.7 \\
\hline & & & & 3 & 80 & 545 & 14.7 \\
\hline & & & & Average & $100 \pm 71$ & $363 \pm 148$ & 27.5 \\
\hline \multirow[t]{15}{*}{ Barley } & $g f p$ & RGEN & pTARGET-gfp2 & 1 & 72 & 207 & 34.8 \\
\hline & & & + RGEN-gfp & 2 & 55 & 206 & 26.7 \\
\hline & & & & 3 & 83 & 255 & 32.5 \\
\hline & & & & Average & $70 \pm 12$ & $223 \pm 23$ & 31.4 \\
\hline & & & pTARGET-gfp2 & 1 & 0 & 46 & 0 \\
\hline & & TALEN & pTARGET-gfp1 & 1 & 71 & 223 & 31.8 \\
\hline & & & + TALEN-gfp & 2 & 110 & 331 & 33.2 \\
\hline & & & & 3 & 49 & 195 & 25.1 \\
\hline & & & & Average & $77 \pm 25$ & $250 \pm 59$ & 30.7 \\
\hline & & & pTARGET-gfp1 & 1 & 0 & 44 & 0 \\
\hline & $M L O$ & TALEN & pTARGET-MLO & 1 & 134 & 350 & 38.3 \\
\hline & & & + TALEN-MLO & 2 & 107 & 254 & 42.1 \\
\hline & & & & 3 & 112 & 237 & 47.3 \\
\hline & & & & Average & $118 \pm 12$ & $280 \pm 50$ & 42.0 \\
\hline & & & PTARGET-MLO & 1 & 0 & 52 & 0 \\
\hline
\end{tabular}

Table 3 Stable transgenic plants expressing RGEN or TALEN constructs

\begin{tabular}{|c|c|c|c|c|c|c|}
\hline \multirow[t]{2}{*}{ Plant species } & \multirow{2}{*}{$\begin{array}{l}\text { Target } \\
\text { gene }\end{array}$} & \multirow{2}{*}{$\begin{array}{l}\text { Type of customized } \\
\text { endonuclease }\end{array}$} & \multicolumn{2}{|c|}{ PCR positive } & \multirow{2}{*}{$\begin{array}{l}\text { Mutant } \\
\text { plants }\end{array}$} & \multirow[t]{2}{*}{ Ratio PCR+/mutants (\%) } \\
\hline & & & Cas9/Fokl & gRNA & & \\
\hline \multirow[t]{2}{*}{ Tobacco } & $g f p$ & RGEN & 17 & 15 & 12 & 80.0 \\
\hline & & TALEN & 35 & n.a. & 1 & 2.9 \\
\hline \multirow[t]{2}{*}{ Barley } & $g f p$ & TALEN & 66 & n.a. & 4 & 6.1 \\
\hline & $M L O$ & TALEN & 6 & n.a. & 3 & 50.0 \\
\hline
\end{tabular}

n.a. not analysed

achieved using the $g f p$-specific TALENs, $80 \%$ of the primary transgenic tobacco plants harboring the $g f p$-specific RGEN-encoding sequence contained indels in the target sequence (Table 3). Nekrasov et al. [29] have reported a mutation frequency of $6.6 \%$ in the $N$. benthamiana PHYTOENE DESATURASE (PDS) sequence by co-expressing Cas 9 and gRNA. At the same time, Li et al. [30] have shown that both the expression level of the gRNA and the size of the ratio of Cas9 to gRNA are important determinants of mutagenic potential. A very high efficiency (>84 \%) has been claimed in RGEN experiments involving N. tabacum PDS or PLEIOTROPIC DRUG RESISTANCE6 (PDR6), in which Cas9 and gRNA were presented within a single expression vector [31]. The efficiency of 


\begin{tabular}{|c|c|c|c|}
\hline GFP-WT & AGGGCGACACCCTGGTGAACCGCATCGAGCTGAAGGGCATCGACTTCAAGGAGGACGGCAACATCCTGGGG & & \\
\hline BM33_639 & 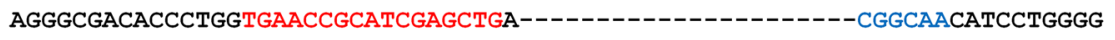 & d22 & $3 / 7$ \\
\hline BM35_697 & 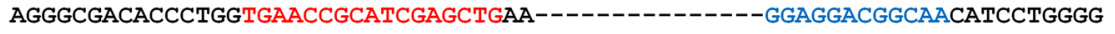 & d15 & $5 / 6$ \\
\hline BM36_757 & AGGGCGACACCCTGGTGAACCGCATCGAGCTGAA-------------GGAGGACGGCAACATCCTGGGG & d15 & $5 / 6$ \\
\hline BM37_760 & $-0-1$ & d172 & $5 / 5$ \\
\hline
\end{tabular}

Fig. 3 Alignment of mutated gfp sequences recovered from four barley transformants (BM33_639, BM35_697, BM36_757, and BM37_760) induced by the presence of a TALEN pair. gfp-specific PCR products were subcloned and up to ten clones were sequenced. The sequences shown in red and blue represent, respectively the left and right TALEN binding sites; the number of nucleotides deleted (dashes) and the number of mutants/number of clones analysed is shown to the right of each sequence

$\begin{array}{llll}\text { WT } & \text { GTCCGTCCTCATGGAACACGGCCTCCACAAGCTCGGCCATGTAAGTCCCGTTACCCTAGCTCAATCAGCA } & \\ & & & \\ 1 \mathrm{E01-1} & \text { GTCCGTCCTCATGGAACACGGCCTCCACAAGCTCG-------TAAGTCCCGTTACCCTAGCTCAATCAGCA } & \text { d6 } & 7 / 10 \\ 1 \mathrm{E} 01-2 & \text { GTCCGTCCTCATGGAACACGGCCTCCACAAGCTC--------AAGTCCCGTTACCCTAGCTCAATCAGCA } & \text { d8 } & 3 / 10 \\ 1 \mathrm{E} 03-7 & \text { GTCCGTCCTCATGGAACACGGCC------------ATGTAAGTCCCGTTACCCTAGCTCAATCAGCA } & \text { d15 } & 4 / 11 \\ 2 \mathrm{E} 02-3 & \text { GTCCGTCCTCATGGAACACGGCCTCCACAAGCTCGGCCA-----GTCCCGTTACCCTAGCTCAATCAGCA } & \text { d5 } & 6 / 9 \\ 2 \mathrm{E} 02-10 & \text { GTCCGTCCTCATGGAACACGGCCTCCACAAGCTCG----TGTAAGTCCCGTTACCCTAGCTCAATCAGCA } & \text { d4 } & 3 / 9\end{array}$

Fig. 4 Alignment of mutated MLO sequences recovered from three barley transformants (1E01, 1E03, and 2E02) induced by the presence of a TALEN construct. MLO-specific PCR products were subcloned and up to ten clones were sequenced. The sequences shown in red and blue represent, respectively the left and right TALEN binding sites; the number of nucleotides deleted (dashes) and the number of mutants/number of clones analysed is shown to the right of each sequence

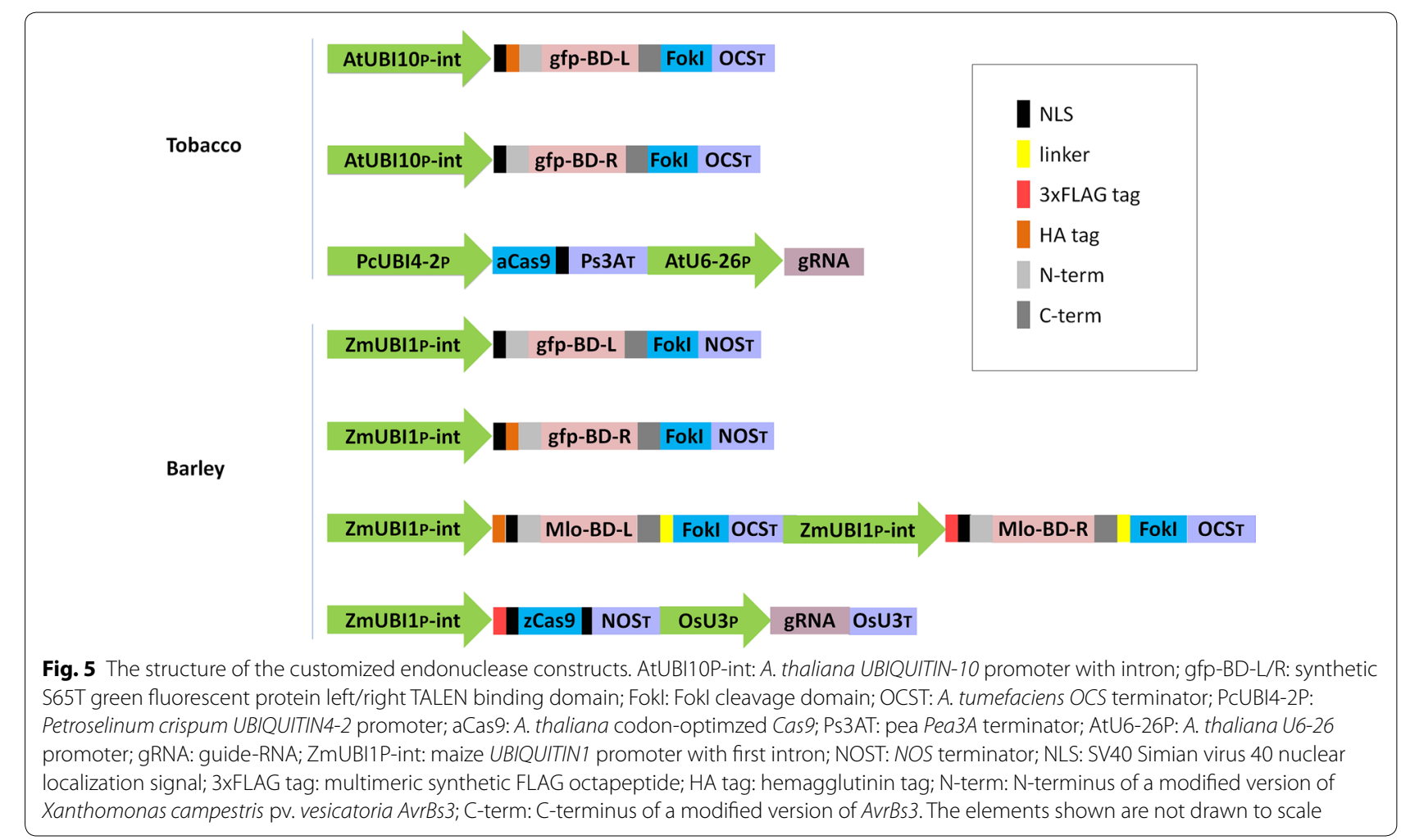

RGEN-mediated mutagenesis is also thought to depend on the accumulation of Cas9 protein. In tobacco, the mutation frequency was reduced when the human codon-optimized variant of Cas9 was employed [29], while Gao et al. [31] used a tobacco codon-optimized version. Here, a single expression vector was designed on the basis of the A. thaliana codon-optimized Cas 9 [32], and this induced a high mutation frequency.

When the system was applied to the more refractory monocotyledonous species barley, the same choice of 
target gene $(g f p)$ was made, since it has been established that $g f p$-specific TALENs are effective inducers of heritable mutations in cultured barley cells [8]. As in tobacco, co-bombardment of pTARGET-gfp1 with the two TALEN units led to the induction of $y f p$ expression in about a third of the red fluorescing cells (Table 2). In contrast to tobacco though, the cleavage activities of the $g f p$-specific TALEN and RGEN constructs were identical (Table 2). The comparatively poor performance of the latter in barley indicates that different target sites may have different accessibility for the endonucleases. The generation of stable $g f p$ mutants via the transformation of immature embryos proved to be less efficient than that achievable from embryogenic pollen cultures [8], possibly because the effectiveness of the maize UBI1 promoter is cell-type dependent. The stable generation of $m l o$ mutants corroborated the high TALEN activity detected in the transient expression assay. The $M L O$-specific TALEN pair was almost as efficient as the $g f p$-specific RGEN construct in tobacco (Table 3 ), clearly showing that a pre-validation of TALEN constructs on the basis of their transient expression is an effective procedure, because only one out of five tested $M L O$-specific TALEN constructs showed activity in a reproducible manner (Additional file 3).

The differences between transient expression and stable transgenic plants may be assigned to the fact that expression strength strongly depends on the number of expression units present within a given cell (gene dosage), the specificity and strength of the promoter driving the transgene, the cell type and developmental stage as well as further conditions, which differ in approaches involving different gene transfer methods and target tissues such as barley and tobacco epidermal cell layers and immature embryos, which were used in the present study. In addition, the $g f p$-TALENs were driven by another $Z m U B I 1$ promoter version than the one used in the $M L O$-TALEN and $g f p$-RGEN constructs.

\section{Conclusions}

In summary, a convenient in planta test system for the cleavage activity of sequence-specifically customized endonucleases was established and exemplified using TALENs and RGENs. It is applicable in both dicot and monocot plant species, which makes it a universal tool for the plant science community. This system may not only facilitate the validation of endonuclease functionality prior to the generation of stable mutant plants but also enable researchers to study general principles of endonuclease activity and to optimize construct design.

\section{Methods}

\section{Plant material}

Four week old seedlings of wild type tobacco ( $N$. tabacum cv. SR1) were used for the transient transgene expression experiments. In the stable transformation experiment, seeds of TSP20L1-1, an established transgenic line harboring a single copy of $g f p$ [15], were surface-sterilized and germinated on [33] solid medium for 2 weeks, after which the seedlings were transferred to culture boxes $(107 \times 94 \times 96 \mathrm{~cm})$ for a further 4 weeks. Barley $(\mathrm{H}$. vulgare) cvs. 'Golden Promise' and 'Ingrid' were used for the transient transgene expression experiments and cv. 'Golden Promise' for the mutation of $M L O$, while a transgenic line of cv. 'Igri' was used for the $g f p$-specific TALEN experiment; the latter's seedlings required vernalization (8 weeks at $4{ }^{\circ} \mathrm{C}$ under a $9 \mathrm{~h}$ photoperiod).

\section{Transient expression test vector construction}

Details of the cloning steps, based on standard procedures, plasmid maps and sequences (Additional file 6), primers and functional elements, are provided in Additional files 4 and 5, and Fig. 5. The generic vector pNB1 (GenBank: KU705395) carries a modified $y f p$ reporter gene [11] driven by a doubled enhanced CaMV $35 S$ promoter [34]; it includes a BamHI and an EcoRI cloning site between the sequences encoding the pea Legumin B4 signal peptide, $y f p$ and a C-terminal KDEL motif for protein retention in the endoplasmic reticulum. Nuclease-specific vectors were developed by inserting $20-50 \mathrm{bp}$ target motifs (annealed oligos, Additional file 4) into the BamHI and EcoRI sites to generate the constructs pTARGETMLO, pTARGET-gfp1 and pTARGET-gfp2 (Additional file 6).

\section{TALEN vector construction}

The $g f p$-specific TALEN vectors [8] comprise a left and a right TALEN unit, each driven by the maize UBIQUITIN1 promoter [35], along with the bialaphos resistanceconferring BAR gene driven by a doubled enhanced $C a M V 35 S$ promoter. The $g f p$-specific TALEN sequences used in the tobacco constructs were identical to those used in barley [8]. However, unlike in the barley constructs, in tobacco the left and right TALEN units were introduced into pUbiAt-OCS (DNA-Cloning-Service, Hamburg, Germany), allowing them to be driven by the A. thaliana UBIQUITIN-10 promoter; their terminal sequence was from the A. tumefaciens OCTOPINE SYNTHASE (OCS) gene. Each TALEN expression cassette was introduced into the p6N vector via its $S f i$ cloning sites, which harbors the HYGROMYCIN PHOSPHOTRANSFERASE (HPT) gene driven by the A. tumefaciens NOPALINE SYNTHASE promoter. The pSP10 (left TALEN unit) and pSP11 (right TALEN unit) vectors (Additional file 6) were introduced into A. tumefaciens strain GV2260 using a heat shock protocol.

The $M L O$-specific TALEN effector binding elements were preceded by a T, $18 \mathrm{bp}$ long, and separated by a 15 
nt spacer sequence (Additional file 1). Repeat lacking TALEN units (pICH47732 TALEN $\Delta$ Rep and pICH47742 TALEN $\Delta$ Rep) were assembled with $B s a$ I site-flanked modules encoding a truncated CaMV $35 S$ promoter (pICH51277, see [36]), HA-NLS [37], a truncated TALE N- and C-terminus, FokI [38] and OCS terminator (pICH41432, [36]) into pICH47732 and pICH47742 [36], respectively. The repeat domains of TALEN 114 and TALEN 115 were created following Morbitzer et al. [39] and were cloned into the Bpil site of pICH47732 TALEN $\triangle$ Rep and pICH47742 TALEN $\triangle$ Rep. TALEN modules with repeats (pICH47732 TALEN 114 and pICH47742 TALEN115) were assembled together with pICH47744 [36] into pUC57_BpiI/KpnI_shuttle via BpiI cut-ligation and thereby flanked by KpnI. Both of the TALEN KpnI fragments were introduced into the KpnI site of the p6int vector (DNA-Cloning-Service, Hamburg, Germany). The TALEN encoding T-DNA vector used for the GUS reporter system was assembled via BpiI cut-ligation from pICH47732 TALEN 114, pICH47742 TALEN 115, pICH47751 Kanamycin, a vector which confers resistance to kanamycin, and pICH47766 [36] into pICH50505 [36] (details and sequences given in Additional file 5).

\section{RGEN vector construction}

To generate a monocotyledonous species-specific generic RGEN vector, two $S f i$ restriction sites were first inserted into pBUN411 [40]. In addition the BAR gene was removed to produce $\mathrm{pSH} 91$. The $g f p$-specific RGEN vector $\mathrm{pSH} 92$ was generated by replacing the spectinomycin resistance gene via $B s a \mathrm{I}$ digestion with a synthetic DNA fragment containing a $g f p$-specific protospacer, formed by annealing the partially complementary oligonucleotides GFP_PP1_f and GFP_PP1_r (Additional file 4). For the tobacco $g f p$-specific RGEN, the Gateway ${ }^{\circledR}$-compatible Cas 9 expression system [32] was used. The above-mentioned $g f p$-specific protospacer sequence was introduced into pEN-Chimera via the pair of BbsI sites. The resulting construct, driven by the $A$. thaliana U6-26 promoter, was then transferred into pDe-CAS9 via a single site Gateway ${ }^{\circledR}$ LR reaction [32], ensuring that Cas 9 lay under the control of the Petroselinum crispum Pclubi 4-2 promoter and the pea Pea3A terminator sequence. The resulting pSI24 vector (Additional file 6) was introduced into $A$. tumefaciens strain GV2260 using a heat shock protocol.

\section{Transient transgenesis via particle bombardment}

Barley and N. tabacum leaf explants were transiently transformed using a PDS-1000/He $\mathrm{Hepta}^{\mathrm{TM}}$ device equipped with a 1100 psi rupture disc (Bio-Rad, Munich, Germany). For barley, six primary leaves harvested from 7 to 8 day old seedlings were placed adaxial side up on
$1 \%$ agar containing $20 \mu \mathrm{g} / \mathrm{mL}$ benzimidazol and $20 \mu \mathrm{g} /$ $\mathrm{mL}$ chloramphenicol. For $N$. tabacum, a single leaf harvested from a 4 week old plant was placed on solidified ( $0.8 \%$ agar) Murashige and Skoog [33] medium containing $2 \%$ sucrose and $400 \mathrm{mg} / \mathrm{L}$ ticarcillin. A $7 \mu \mathrm{g}$ aliquot of plasmid DNA was mixed with $3 \mathrm{mg}$ gold micro-carriers by vortexing in the presence of $25 \mu \mathrm{L} 25 \mathrm{mM} \mathrm{CaCl}_{2}$ and $10 \mu \mathrm{L} 0.1 \mathrm{M}$ spermidine. After centrifugation, the pellet was washed with 75 and $100 \%$ ethanol, followed by suspension in $60 \mu \mathrm{L} 100 \%$ ethanol. A total of $4 \mu \mathrm{L}$ of coated micro-carrier suspension was loaded onto each of the seven macro-carriers, as recommended by the PDS1000/He manual. Each set of explants were bombarded twice with a total amount of 16-19 $\mu$ g plasmid DNA ( $7 \mu \mathrm{g}$ nuclease test vector, $7 \mu \mathrm{g}$ endonuclease vector and 2-5 $\mu \mathrm{g}$ mCherry vector), then incubated at room temperature for 1 day before assaying for fluorescence. Each experiment was carried out three times.

\section{Transient transgenesis via Agrobacterium tumefaciens infiltration}

The A. tumefaciens strain GV3101 pMP90RK [41] was used for transient expression assays in $N$. benthamiana. Liquid culture-grown $\left(28{ }^{\circ} \mathrm{C}, 180 \mathrm{rpm}\right.$ for 1 day) Agrobacterium was set to an $\mathrm{OD}_{600}$ of 1.0 with infiltration solution (10 mM $\mathrm{MgCl}_{2}, 10 \mathrm{mM} \mathrm{MES} \mathrm{(pH} \mathrm{5.7),}$ $200 \mu \mathrm{M}$ acetosyringone). The bacteria were delivered into 3-4 week-old $N$. benthamiana leaves using a needleless syringe. After 2 days, infiltrated areas were cut out and de-stained in $80 \%(\mathrm{v} / \mathrm{v})$ ethanol for a few days prior to GUS staining (see below).

\section{Stable transformants of barley and tobacco}

Barley was transformed according to Hensel et al. [42], except that the immature embryos harvested from transgenic single-copy, $g f p$ expressing cv. 'Igri' were initially cultured for 5 days on BPCM (solid BCIM, $5 \mathrm{mg} / \mathrm{L}$ dicamba) before the introduction of $A$. tumefaciens. Tobacco ( $N$. tabacum wild type or line TSP20L1-1) plants were transformed with pGH292 or pSI24, respectively. This vector harbors $g f p$ controlled by the $A$. thaliana UBIQUITIN-10 promoter and the A. tumefaciens OCS terminator. The NEOMYCIN PHOSPHOTRANSFERASE gene (NptII) for kanamycin resistance in planta is driven by the CaMV $35 S$ promoter and termination sequence. The transgene was introduced into $A$. tumefaciens strain GV2260 using a heat shock protocol. Leaf sections $\left(\sim 1 \mathrm{~cm}^{2}\right)$ excised from sterile-grown plants were laid on Murashige and Skoog [33] medium containing $3 \% \mathrm{w} / \mathrm{v}$ sucrose, $1 \mathrm{mg} / \mathrm{L}$ 6-benzylaminopurine, $0.1 \mathrm{mg} / \mathrm{L}$ 1-naphthalene acetic acid and 2\% agar for 1-2 days, before inoculation with the transgenic A. tumefaciens for $30 \mathrm{~min}$. The explants were blotted with sterile filter paper 
and kept for 3 days at $19{ }^{\circ} \mathrm{C}$ in the dark, on medium supplemented with $400 \mathrm{mg} / \mathrm{L}$ ticarcillin and either $100 \mathrm{mg} / \mathrm{L}$ kanamycin or $5 \mathrm{mg} / \mathrm{L}$ bialaphos at $22^{\circ} \mathrm{C}$. Developing calli were sub-cultured every 10 days. After emergence of first shoots, the plates were transferred to light ( $16 \mathrm{~h}$ photoperiod) until shoots had reached $1 \mathrm{~cm}$ in length, at which point these were excised and placed on Murashige and Skoog [33] medium containing $2 \% \mathrm{w} / \mathrm{v}$ sucrose, $0.8 \%$ agar to stimulate root initiation. Plantlets which had developed a viable root system were transferred to the greenhouse.

\section{Genomic DNA isolation and PCR}

Genomic DNA was isolated from snap-frozen leaves following Palotta et al. [43]. Subsequent $20 \mu \mathrm{L}$ PCRs were formulated with 50-100 ng template DNA, and primed as listed in Additional file 4. The reaction products were purified using a QIAquick PCR Purification kit (Qiagen, Hilden, Germany) to allow for amplicon sequencing. Target-specific PCR products amplified from transgenic individuals were cloned into pGEM-T Easy (Promega, Mannheim, Germany). After blue-white selection, plasmid DNA was isolated from ten positive clones and sequenced.

\section{Confocal microscopy}

Frequency of TALEN or RGEN construct induced mutations was determined from the ratio between the number of yellow-fluorescent (YFP) and red-fluorescent (mCherry) cells. For this a total of six leaves of barley and one leaf of $N$. tabacum were analyzed with a Zeiss LSM780 confocal laser microscope (Carl Zeiss, Jena, Germany). YFP fluorescence was visualized using a $514 \mathrm{~nm}$ laser line in combination with a 517-560 nm bandpass; mCherry fluorescence was visualized with a $561 \mathrm{~nm}$ laser line in combination with a $570-620 \mathrm{~nm}$ bandpass.

\section{GUS staining}

Barley and $N$. benthamiana leaves were harvested for GUS staining three and 2 days, respectively, after bombardment/or Agrobacterium infiltration. The leaves were submerged in 5-bromo-4-chloro-3-indolyl-B-D-glucoronide cyclohexylammonium (X-Gluc) staining solution (42.3 $\mathrm{mM} \mathrm{NaH}{ }_{2} \mathrm{PO}_{4}, 57.7 \mathrm{mM} \mathrm{Na} \mathrm{HPO}_{4}, 10 \mathrm{mM}$ EDTA, $20 \%$ methanol, $5 \mathrm{mM} \mathrm{K}_{3} \mathrm{Fe}(\mathrm{CN})_{6}, 5 \mathrm{mM} \mathrm{K}_{4} \mathrm{Fe}(\mathrm{CN})_{6} \times 3$ $\mathrm{H}_{2} \mathrm{O}, 1 \mathrm{mg} / \mathrm{mL}$ X-Gluc, $0.1 \%$ Triton X-100) and vacuum was applied three times for $10 \mathrm{~min}$. Then, the material was incubated at $37{ }^{\circ} \mathrm{C}$ overnight. Afterwards, the leaves were bleached in $80 \% \mathrm{EtOH}$ at room temperature for at least 2 days. The leaves were screened for blue-stained cells by bright field microscopy; photographs were taken with the Keyence Biorevo BZ9000 microscope (Keyence Corporation, Neu-Isenburg, Germany).

\section{Additional files}

Additional file 1. MLO-specific TALEN target sequences.

Additional file 2. (a) Step-wise functional principle of transient expression vector system for assessing the relative cleavage activity of customized endonucleases. Incorporation of a target site for sequencespecific endonucleases deliberately generates a frame shift in the codon sequence of GUS. Upon co-transformation of target vector along with respective customized TALENs, double-strand breaks at the target site are induced. The repair of double-strand breaks at target site via non-homologous end-joining, which often introduces indels, render GUS back in frame and GUS protein can be detected by X-Gluc staining. (b) Example for successful employment of the reporter system in a transient assay in barley. From left to right: negative control (reporter construct only) and two examples of successful induction of indels indicated by blue-green GUS staining of the cell. Upper panel: barley (Hordeum vulgare cv. 'Ingrid', Hv) after bombardment; lower panel: $N$. benthamiana (Nb) after Agrobacterium infiltration. Arrows highlight some GUS-stained cells. Bars: 50 um (upper panel); $100 \mu \mathrm{m}$ (lower panel).

Additional file 3. Transient expression test of MLO-specific TALEN constructs in barley (using bombardment) and N. benthamiana (using agroinfection).

Additional file 4. Oligomers used to clone PTARGET and RGEN plasmids, and the PCR-based analysis of putative transgenic regenerants.

Additional file 5. Details and sequences for the MLO-specific TALEN constructs.

Additional file 6. Plasmid maps and sequences of the constructs.

\section{Authors' contributions}

JK, GH, TL and RP conceived the study. NB, SS, SP, MG, SH, SK, RM, TR and GH generated the necessary plasmids, performed the experiments and analysed the data. NB, SS, JK and GH wrote the manuscript. All authors read and approved the final manuscript.

\section{Author details}

1 Plant Reproductive Biology, Leibniz Institute of Plant Genetics and Crop Plant Research (IPK), Corrensstr. 3, 06466 Stadt Seeland/OT Gatersleben, Germany. ${ }^{2}$ Present Address: Chair of Plant Breeding, Martin Luther University, Betty-Heimann-Str. 3, 06120 Halle (Saale), Germany. ${ }^{3}$ Structural Cell Biology, Leibniz Institute of Plant Genetics and Crop Plant Research (IPK), Corrensstr. 3, 06466 Stadt Seeland/OT Gatersleben, Germany. ${ }^{4}$ Unit of Plant Molecular Cell Biology, Institute for Biology I, RWTH Aachen University, Worringerweg 1 , 52056 Aachen, Germany. ${ }^{5}$ ZMBP-General Genetics, University of Tübingen, Auf der Morgenstelle 32, 72076 Tübingen, Germany.

\section{Acknowledgements}

We appreciate the excellent technical assistance given by Sabine Sommerfeld, Sibylle Freist and Petra Hoffmeister. The research aimed at establishing TALENs as a tool for genome editing in plants is supported by a DFG Grants to TL (LA 1338/5-1). The Gateway ${ }^{\circledR}$-compatible Cas9 expression system was kindly provided by H. Puchta (KIT, Karlsruhe, Germany).

\section{Competing interests}

The authors declare that they have no competing interests.

Received: 7 January 2016 Accepted: 24 February 2016

Published online: 09 March 2016 


\section{References}

1. CermakT, Doyle EL, Christian M, Wang L, Zhang Y, Schmidt C, Baller JA, Somia NV, Bogdanove AJ, Voytas DF. Efficient design and assembly of custom TALEN and other TAL effector-based constructs for DNA targeting. Nucleic Acids Res. 2011;39:e82.

2. Li T, Liu B, Spalding MH, Weeks DP, Yang B. High-efficiency TALEN based gene editing produces disease-resistant rice. Nat Biotechnol. 2012;30:390-2.

3. Shan Q, Wang Y, Li J, Zhang Y, Chen K, Liang Z, Zhang K, Liu J, Xi JJ, Qiu J-L, Gao C. Targeted genome modification of crop plants using a CRISPR-Cas system. Nat Biotechnol. 2013;31:686-8.

4. Zetsche B, Gootenberg JS, Abudayyeh OO, Slaymaker IM, Makarova KS, Essletzbichler P, Volz SE, Joung J, van der Oost J, Regev A, Koonin EV, Zhang F. Cpf1 Is a single RNA-guided endonuclease of a class 2 CRISPRCas system. Cell. 2015;163:759-71.

5. Puchta H, Dujon B, Hohn B. Two different but related mechanisms are used in plants for the repair of genomic double-strand breaks by homologous recombination. Proc Natl Acad Sci USA. 1996;93:5055-60.

6. Jasin M, Rothstein R. Repair of strand breaks by homologous recombination. Cold Spring Harb Perspect Biol. 2013;5:a012740.

7. Baltes NJ, Voytas DF. Enabling plant synthetic biology through genome engineering. Trends Biotechnol. 2015;33:120-31.

8. Gurushidze M, Hensel G, Hiekel S, Schedel S, Valkov V, Kumlehn J. Truebreeding targeted gene knock-out in barley using designer TALE-nuclease in haploid cells. PLoS ONE. 2014;9:e92046.

9. Lawrenson T, Shorinola O, Stacey N, Li C, Ostergaard L, Patron N, Uauy C, Harwood W. Induction of targeted, heritable mutations in barley and Brassica oleracea using RNA-guided Cas9 nuclease. Genome Biol. 2015;16:258.

10. Wang YP, Cheng X, Shan QW, Zhang Y, Liu JX, Gao CX, Qiu JL. Simultaneous editing of three homoeoalleles in hexaploid bread wheat confers heritable resistance to powdery mildew. Nat Biotechnol. 2014;32:947-51.

11. Budhagatapalli N, Rutten T, Gurushidze M, Kumlehn J, Hensel G. Targeted modification of gene function exploiting homology-directed repair of TALEN-mediated double-strand breaks in barley. G3 Genes Genomes Genet. 2015;5:1857-63.

12. Kim H, Kim JS. A guide to genome engineering with programmable nucleases. Nat Rev Genet. 2014;15:321-34

13. Buschges $R$, Hollricher $K$, Panstruga $R$, Simons $G$, Wolter M, Frijters $A$, van Daelen R, van der Lee T, Diergaarde P, Groenendijk J, Toepsch S, Vos R, Salamini F, Schulze-Lefert P. The barley MLO gene: a novel control element of plant pathogen resistance. Cell. 1997;88:695-705.

14. Piffanelli P, Ramsay L, Waugh R, Benabdelmouna A, D'Hont A, Hollricher $\mathrm{K}$, Jorgensen JH, Schulze-Lefert P, Panstruga R. A barley cultivation-associated polymorphism conveys resistance to powdery mildew. Nature. 2004:430:887-91.

15. Schedel S, Pencs S, Hensel G, Mueller A, Kumlehn J. RNA-guided endonuclease-driven mutagenesis in tobacco followed by efficient fixation of mutated sequences in doubled haploid plants. doi:10.1101/042291

16. Target Finder. https://tale-nt.cac.cornell.edu. Accessed 7 Jan 2016.

17. Perez-Quintero A, Rodriguez-R LM, Dereeper A, Lopez C, Koebnik R, Szurek B, Cunnac S. An improved method for TAL effectors DNA-binding sites prediction reveals functional convergence in TAL repertoires of Xanthomonas oryzae strains. PLoS ONE. 2013;8:e68464.

18. Talvez. http://bioinfo.mpl.ird.fr/cgi-bin/talvez/talvez.cgi. Accessed 7 Jan 2016

19. TALgetter. http://galaxy2.informatik.uni-halle.de:8976/tool_runner?tool_ id=TALgetter. Accessed 7 Jan 2016.

20. CRISPR design. http://crispr.mit.edu/. Accessed 7 Jan 2016.

21. CRISPRer. http://galaxy2.informatik.uni-halle.de:8976/. Accessed 7 Jan 2016

22. Deskgen. https://www.deskgen.com/landing/. Accessed 7 Jan 2016

23. Noel ES, Verhoeven M, Lagendijk AK, Tessadori F, Smith K, Choorapoikayil $\mathrm{S}$, den Hertog J, Bakkers J. A Nodal-independent and tissue-intrinsic mechanism controls heart-looping chirality. Nat Commun. 2013;4:2754.
24. Jiang W, Zhou H, Bi H, Fromm M, Yang B, Weeks DP. Demonstration of CRISPR/Cas9/sgRNA-mediated targeted gene modification in Arabidopsis, tobacco, sorghum and rice. Nucleic Acid Res. 2013;41(20):e188.

25. Yin K, Han T, Liu G, Chen T, Wang Y, Yu AYL, Liu Y. A geminivirus-based guide RNA delivery system for CRISPR/Cas9 mediated plant genome editing. Sci Rep. 2015;5:14926.

26. Panstruga R, Kim MC, Cho MJ, Schulze-Lefert P. Testing the efficiency of dsRNAi constructs in vivo: a transient expression assay based on two fluorescent proteins. Mol Biol Rep. 2003;30:135-40.

27. Zhang H, Zhang JS, Wei PL, Zhang BT, Gou F, Feng ZY, Mao YF, Yang L, Zhang $\mathrm{H}$, Xu NF, Zhu JK. The CRISPR/Cas9 system produces specific and homozygous targeted gene editing in rice in one generation. Plant Biotechnol J. 2014;12:797-807.

28. Feng Z, Mao Y, Xu N, Zhang B, Wei P, Yang DL, Wang Z, Zhang Z, Zheng R, Yang $L$, Zeng $L$, Liu $X$, Zhu JK. Multigeneration analysis reveals the inheritance, specificity, and patterns of CRISPR/Cas-induced gene modifications in Arabidopsis. Proc Natl Acad Sci USA. 2014;111:4632-7.

29. Nekrasov V, Staskawicz B, Weigel D, Jones JDG, Kamoun S. Targeted mutagenesis in the model plant Nicotiana benthamiana using Cas9 RNAguided endonuclease. Nat Biotechnol. 2013;31:691-3.

30. Li W, Teng F, Li T, Zhou Q. Simultaneous generation and germline transmission of multiple gene mutations in rat using CRISPR-Cas systems. Nat Biotechnol. 2013;31:684-6.

31. Gao J, Wang G, Ma S, Xie X, Wu X, Zhang X, Wu Y, Zhao P, Xia Q. CRISPR/ Cas9-mediated targeted mutagenesis in Nicotiana tabacum. Plant Mol Biol. 2015;87:99-110.

32. Fauser F, Schiml S, Puchta H. Both CRISPR/Cas-based nucleases and nickases can be used efficiently for genome engineering in Arabidopsis thaliana. Plant J. 2014;79:348-59.

33. Murashige T, Skoog F. A revised medium for rapid growth and bio assays with tobacco tissue cultures. Physiol Plant. 1962;15:473-97.

34. Odell JT, Nagy F, Chua NH. Identification of DNA-sequences required for activity of the cauliflower mosaic virus-35S promoter. Nature. 1985;313:810-2

35. Christensen $\mathrm{AH}$, Quail PH. Ubiquitin promoter-based vectors for highlevel expression of selectable and/or screenable marker genes in monocotyledonous plants. Transgenic Res. 1996;5:213-8.

36. Weber E, Engler C, Gruetzner R, Werner S, Marillonnet S. A modular cloning system for standardized assembly of multigene constructs. PLOS ONE. 2011:6:e16765.

37. de Lange O, Wolf C, Dietze J, Elsaesser J, Morbitzer R, Lahaye T. Programmable DNA-binding proteins from Burkholderia provide a fresh perspective on the TALE-like repeat domain. Nucleic Acids Res. 2014;42:7436-49.

38. Mussolino C, Morbitzer R, Lutge F, Dannemann N, Lahaye T, Cathomen T. A novel TALE nuclease scaffold enables high genome editing activity in combination with low toxicity. Nucleic Acids Res. 2011;39:9283-93.

39. Morbitzer R, Elsaesser J, Hausner J, Lahaye T. Assembly of custom TALEtype DNA binding domains by modular cloning. Nucleic Acids Res. 2011;39:5790-9.

40. Xing HL, Dong L, Wang ZP, Zhang HY, Han CY, Liu B, Wang XC, Chen QJ. A CRISPR/Cas9 toolkit for multiplex genome editing in plants. BMC Plant Biol. 2014;14:327.

41. Koncz C, Schell J. The promoter of T-DNA gene 5 controls the tissue-specific expression of chimaeric genes carried by a novel type of Agrobacterium binary vector. Mol Gen Genet. 1986;204:383-96.

42. Hensel G, Kastner C, Oleszczuk S, Riechen J, Kumlehn J. Agrobacteriummediated gene transfer to cereal crop plants: current protocols for barley, wheat, triticale, and maize. Int J Plant Genomics. 2009;2009:835608.

43. Pallotta MA, Graham RD, Langridge P, Sparrow DHB, Barker SJ. RFLP mapping of manganese efficiency in barley. Theor Appl Genet. 2000;101:1100-8. 